\title{
Toward synthesis of third-generation spin-labeled podophyllotoxin derivatives using isocyanide multicomponent reactions
}

\author{
Liang Kou ${ }^{\mathrm{a}, \dagger}$, Mei-Juan Wang ${ }^{\mathrm{a}, \dagger}$, Li-Ting Wang ${ }^{\mathrm{b}}$, Xiao-Bo Zhao $^{\mathrm{a}}$, Xiang Nan $^{\mathrm{a}}$, Liu Yang ${ }^{\mathrm{d}}$, \\ Ying-Qian Liu ${ }^{1, e,}{ }^{*}$, Susan L. Morris-Natschke ${ }^{b}$, and Kuo-Hsiung Lee ${ }^{b, c,{ }^{*}}$ \\ aSchool of Pharmacy, Lanzhou University, Lanzhou 730000, PR China \\ bNatural Products Research Laboratories, UNC Eshelman School of Pharmacy, University of \\ North Carolina, Chapel Hill, North Carolina 27599 \\ ${ }^{c}$ Chinese Medicine Research and Development Center, China Medical University and Hospital, \\ Taichung, Taiwan \\ dEnvironmental and Municipal Engineering School, Lanzhou Jiaotong University, Lanzhou \\ 730000, PR China \\ eXinjiang Production \& Construction Corps Key Laboratory of Protection and Utilization of \\ Biological Resources in Tarim Basin
}

\begin{abstract}
Spin-labeled podophyllotoxins have elicited widespread interest due to their far superior antitumor activity compared to podophyllotoxin. To extend our prior studies in this research area, we synthesized a new generation of spin-labeled podophyllotoxin analogs via isocyanide multicomponent reactions and evaluated their cytotoxicity against four human cancer cell lines (A-549, DU-145, KB and KBvin). Most of the compounds exhibited potent cytotoxic activity against all four cell lines, notably against the drug resistant KBvin cancer cell line. Among the new analogs, compounds $12 \mathrm{e}\left(\mathrm{IC}_{50}: 0.60-0.75 \mu \mathrm{M}\right)$ and $\mathbf{1 2 h}\left(\mathrm{IC}_{50}: 1.12-2.03 \mu \mathrm{M}\right)$ showed superior potency to etoposide ( $\mathrm{IC}_{50}: 2.03->20 \mu \mathrm{M}$ ), a clinically available anticancer drug. With a concise efficient synthesis and potent cytotoxic profiles, compounds $\mathbf{1 2 e}$ and $\mathbf{1 2 h}$ merit further development as a new generation of epipodophyllotoxin-derived antitumor clinical trial candidates.
\end{abstract}

\section{Keywords}

Podophyllotoxin; C-4 position; Spin labeled; Isocyanide multicomponent reactions; Cytotoxic activity

\footnotetext{
(C) 2014 Elsevier Masson SAS. All rights reserved.

”To whom correspondence should be addressed. Tel.: (919) 962-0066. Fax: (919) 966-3893. khlee@ email.unc.edu (K.H. Lee); yqliu@1zu.edu.cn (Y.Q. Liu).

These authors contributed equally to this work.

Publisher's Disclaimer: This is a PDF file of an unedited manuscript that has been accepted for publication. As a service to our customers we are providing this early version of the manuscript. The manuscript will undergo copyediting, typesetting, and review of the resulting proof before it is published in its final citable form. Please note that during the production process errors may be discovered which could affect the content, and all legal disclaimers that apply to the journal pertain.
} 


\section{Introduction}

In recent years, multi-component reactions (MCRs) have played a central role in the development of modern synthetic methodology for drug discovery research. The applications in this field are quite broad extending from initial lead structure identification to generation of large libraries of analogs. Combined with the power of in silico screens, this methodology is an efficient tool for the modern medicinal chemist. MCRs that involve isocyanides (IMCRs) are by far the most versatile reactions in terms of scaffolds and number of accessible compounds [1-3]. Many advantages over conventional linear-type syntheses make IMCRs very popular in the medicinal chemistry community: superior atom economy, low costs, high variability, high bond forming efficiency, and simple environmentally benign procedures. From this standpoint, design- or logic-based discovery utilizing IMCR could be advantageous. Consequently, the application of IMCR offers an efficient synthetic route to construct diverse podophyllotoxin-based scaffolds for drug discovery.

Podophyllotoxin (1, PPT), a naturally occurring lignin, exerts cytotoxic activity by inhibition of microtubule assembly [4,5]. Its two semisynthetic glucoconjugates, etoposide (2) and teniposide (3), are novel DNA topoisomerase II inhibitors marketed in several countries $[6,7]$. Some non-sugar substituted analogues, particularly $N$-linked congeners, exhibit superior pharmacological properties to etoposide, and consequently, several newergeneration clinical candidates, including NPF (4) [8], GL-331 (5) [9], and TOP-53 (6) [10], have emerged through C-4 modification as alternatives to overcome the drawbacks of etoposide. In addition, another important area of podophyllotoxin research involves the synthesis and design of novel spin-labeled PPT derivatives by our group over the past decades [11-20], providing potential drugs with beneficial therapeutic profiles, e.g., improved activity or higher solubility, better pharmacokinetics, or reduced side effects. Among the highlights of this research, first generation spin-labeled podophyllotoxin derivatives represented by GP-7 (7, Fig. 1) and GP-11 (8) are the most important, because of their high potency, low toxicity, broad antitumor spectra, and better formulation/usage characteristics. After them, second generation spin-labeled amino acid-linked derivatives were investigated [21-23] and some of them showed superior or comparable activity against particular cancer types compared to 2 , while their drug-resistance profiles were significantly different from those of $\mathbf{1}$. Besides, some compounds were effective at a lower dose than etoposide in drug-sensitive and drug-resistant xenograft models, demonstrating their potential as drug candidates for anticancer chemotherapy.

Although numerous syntheses and structure modifications of podophyllotoxins have been performed, most changes at the C-4 position of PPT have been relatively simple, such as incorporation of $4 \beta$-substituted ethers, esters, and $N$-linked congeners [24-30]. Modification of this position with diverse structures via MCRs has attracted less interest. Furthermore, typical synthetic strategies often suffer from one or more limitations, including long reaction times, tedious work-up procedures, or lack of general applicability and stereoselectivity, which makes them unsuitable not only for large-scale synthesis of this class of biologically active molecules but also for synthesis of screening libraries for drug discovery or for industrial production. To enrich the limited set of podophyllotoxin derivatives typically employed in drug discovery, we recently described an efficient synthesis of $4 \beta$ isocyanopodophyllotoxins by dehydration of the corresponding formamide under both ultrasonic and classical conditions [31]. Based on the success of the above reaction, we subsequently developed a practical IMCR to synthesize podophyllotoxin derivatives. Herein, we first report the use of Ugi (Ugi-4CR) and Passirini (P-3CR) reactions as a versatile approach towards the synthesis of third generation novel spin-labeled podophyllotoxin series. To our knowledge, these examples are also the first where a nitroxide participated in 
such IMCRs. Some of the obtained compounds have been screened for cytotoxic activity and exhibited considerable, yet selective potency against certain cancer cell types. With a concise efficient synthesis and potent cytotoxic profiles, compounds $12 \mathrm{e}, 12 \mathrm{~h}$ and $13 \mathrm{c}$ merit further development as new generation spin-labeled epipodophyllotoxin-derived antitumor clinical trial candidates.

\section{Results and discussion}

\subsection{Chemistry}

Our synthetic routes are outlined in Schemes 1 and 2. Briefly, the precursor $4 \beta$ aminoepipodophyllotoxin (9) was prepared stereoselectively from $\mathbf{1}$ through azidation and catalytic hydrogenation according to a previously reported method [32]. Next, compound 9 was converted to $4 \beta$-formamido-4-deoxypodophyllotoxin (10) in excellent yield by reaction with ethyl formate under both classical and ultrasonic conditions. Subsequently, dehydration of $\mathbf{1 0}$ with $p$-toluenesulfonyl chloride in pyridine furnished the key intermediate $4 \beta$ isocyanopodophyllotoxin (11) in good yield [31]. Finally, two novel series of target compounds 12a-j and 13a-l were obtained via Passerini and Ugi reactions in moderate to good yields, respectively. The Passirini reaction (Scheme 1) involved three components: aldehyde/ketone, stable nitroxyl radical acid, and $4 \beta$-isocyanopodophyllotoxin (11). The reaction gave a series of novel spin-labeled epipodophyllotoxin derivatives with an $\alpha$ acyloxy carboxamide group.

The Ugi reaction (Scheme 2) involved four components: aldehyde/ketone, amine, stable nitroxide radical acid, $4 \beta$-isocyanopodophyllotoxin (11). This reaction mixture gave another series of spin-labeled derivatives with a a-acylamino carboxamide group. All newly synthesized compounds were purified by column chromatography and their structures were characterized by HRMS, ESR and IR spectroscopic analysis.

\subsection{Cytotoxicity}

Target compounds 12a-j and 13a-l were evaluated for in vitro cytotoxicity against four human tumor cell lines, A549 (non-small cell lung cancer), DU145 (prostate cancer cell line), KB (nasopharyngeal carcinoma), and KBvin [multi-drug resistant (MDR) KB subline selected using vincristine], using a sulforhodamine B colorimetric (SRB) assay with triplicate experiments [33]. Compound $\mathbf{2}$ was included as a positive control and the results are summarized in Table 1.

As illustrated in Table 1, except for compounds 12b and 13d-l, most of the tested compounds exhibited moderate to potent cytotoxicity against the four human tumor cell lines. Remarkably, these compounds retained similar levels of cytotoxicity against the drug resistant KBvin and KB tumor cell lines, while 2 lost its activity completely $\left(\mathrm{IC}_{50}>20\right.$ vs $3.88 \mu \mathrm{M})$. This result agrees with our prior observation that $\mathrm{C} 4$-amino substitution of epipodophyllotoxin is good for overcoming drug-resistance [34-36]. Among the tested compounds, compounds $12 \mathrm{e}$ and $\mathbf{1 2 h}$ showed superior activity with $\mathrm{IC}_{50}$ values of 0.60 $1.46 \mu \mathrm{M}$ compared to those of $2\left(\mathrm{IC}_{50} 2.03-3.88 \mu \mathrm{M}\right)$ against A549, DU-145, and $\mathrm{KB}$ tumor cell lines.

Compounds 12e and $12 \mathrm{~h}$ have a methoxy and methylenedioxy substituted phenyl ring, respectively, in their a-acyloxy carboxamide side chain, while $\mathbf{1 2 b}$ has an unsubstituted phenyl ring in the same position. This minor structural difference is notable as $\mathbf{1 2 b}$ was completely inactive against all four tumor cell lines. Two additional compounds with an aromatic ring in the a-acyloxy carboxamide side chain, $\mathbf{1 2} \mathbf{g}$ with a furan ring and $\mathbf{1 2 f}$ with a benzyl group, displayed moderate potency $\left(\mathrm{IC}_{50} 6.53-7.71 \mu \mathrm{M}\right)$. Therefore, future structural 
modifications will focus on introducing various substituted aromatic rings in the a-acyloxy carboxamide side chain.

Among compounds 12a, 12c, and 12d with alkyl groups in the a-acyloxy carboxamide side chain, cytotoxic activity generally decreased as the size of the substituents increased. For example, against the A549 cancer cell, the $\mathrm{IC}_{50}$ value of $\mathbf{1 2 a}$ (two methyl groups) was 2.38 $\mu \mathrm{M}$, while the $\mathrm{IC}_{50}$ values of $\mathbf{1 2 c}$ (H and propyl group) and $\mathbf{1 2 d}$ (cyclohexane) were 8.86 and $9.52 \mu \mathrm{M}$, respectively, indicating that the size of substituent groups might critical.

Compounds $\mathbf{1 2 c}, \mathbf{1 2} \mathbf{j}$ and $\mathbf{1 2} \mathbf{i}$ differ only in the identity of the nitroxide. The three compounds exhibited similar potency; thus, ring size and degree of unsaturation had no effect, which was consistent with the literature [19].

Surprisingly, in the a-acylamino carboxamide series, only three (13a-c) compounds displayed cytotoxicity, albeit only moderate, against the four human tumor cell lines. It is not clear why most a-acylamino carboxamide analogs were inactive, while corresponding $a-$ acyloxy carboxamide derivatives (e.g., $13 \mathrm{~h}$ vs $12 \mathrm{e}, \mathbf{1 3 d}$ vs 12a) were active. However, a general relationship between substituent size and activity appears to be present and merits further investigation.

\section{Conclusion}

In summary, efficient isocyanide multicomponent reactions have been developed to prepare newer-generation structurally diverse spin-labeled derivatives of epipodophyllotoxin. This approach is a valuable tool in design and synthesis of new podophyllotoxin analogs with advantages of simplicity, atom-economy, and good yields. The cytotoxic results showed that most of the new compounds exhibited moderate to potent cytotoxic activity against A-549, DU-145, KB and KBvin, and overcame acquired drug resistance in the latter cell line. Among them, compounds $\mathbf{1 2} \mathbf{e}$ and $\mathbf{1 2 h}$ were the most promising derivatives and were selected as lead molecules for further development. Additional systematic structural modifications will be carried out to further clarify these initial interesting findings. Future applications could also involve development of versatile biologically significant podophyllotoxin fragments into MCR products, chemical libraries created via MCRs, and heterocycles built from isocyanides.

\section{Experimental section}

\subsection{Chemistry}

Melting points were determined on a Kofler apparatus and are uncorrected. IR spectra were measured on a Nicolet 380 FT-IR spectrometer on neat samples placed between KBr plates. Mass spectra were recorded on a Bruker Daltonics APEXII49e spectrometer with ESI ionization source. Electron spin resonance (ESR) spectra were obtained on a Bruker A300 $\mathrm{X}$-band EPR spectrometer. ${ }^{1} \mathrm{H}$ and ${ }^{13} \mathrm{C}$ NMR spectra were recorded at $400 \mathrm{MHz}$ and 100 MHz on a BrukerAM-400 spectrometer using TMS as reference (Bruker Company, USA). Podophyllotoxin (1) was isolated from the Chinese medicinal herb Juniperus sabina Linnaeus, and served as the starting material for preparation of all new derivatives. The precursor $4 \beta$-aminoepipodophyllotoxin (9) was synthesized by our previously reported procedures, and its structure was confirmed by direct comparison with an authentic sample and previously reported spectroscopic data [32].

\subsection{Synthesis of key intermediate $4 \beta$-isocyanopodophyllotoxin (11)}

The key intermediate $4 \beta$-isocyanopodophyllotoxin (11) used for the experiments was prepared by our previous procedure [31].Yield 67\%; m.p. $115-117^{\circ} \mathrm{C}$; IR $\left(\mathrm{KBr}, \mathrm{cm}^{-1}\right) 2130$ 
(NC), 1779 (lactone), 1588, 1506 and 1485 (aromatic $\mathrm{C}=\mathrm{C}), 930\left(\mathrm{OCH}_{2} \mathrm{O}\right),{ }^{1} \mathrm{H}$ NMR (400 $\mathrm{MHz}, \mathrm{CDCl}_{3}$ ) $\delta 6.91$ (s, 1H, H-5), 6.56 (s, 1H, H-8), 6.26 (s, 2H, H-2',6'), 6.03 (dd, J = 7.9, $\left.1.1 \mathrm{~Hz}, 2 \mathrm{H}, \mathrm{OCH}_{2} \mathrm{O}\right), 4.99(\mathrm{~d}, J=4.4 \mathrm{~Hz}, 1 \mathrm{H}, \mathrm{H}-4), 4.68(\mathrm{~d}, J=5.1 \mathrm{~Hz}, 1 \mathrm{H}, \mathrm{H}-1), 4.39$ (m, $2 \mathrm{H}, \mathrm{H}-11), 3.81\left(\mathrm{~s}, 3 \mathrm{H}, 4^{\prime}-\mathrm{OCH}_{3}\right), 3.75\left(\mathrm{~s}, 6 \mathrm{H}, 3^{\prime}, 5\right.$ '- $\left.\mathrm{OCH}_{3}\right), 3.20$ (q, 1H, H-2), $2.96(\mathrm{~m}, 1 \mathrm{H}$, H-3). ${ }^{13}$ C NMR: $\left(\mathrm{CDCl}_{3}, 400 \mathrm{MHz}\right) 8: 173.01,160.01,152.78,149.27,147.95,137.64$, $134.32,131.59,125.13,110.53,108.71,108.33,101.95,67.69,60.73,56.35,53.53,43.53$, 41.37, 35.24. MS (EI) $m / z: 424(\mathrm{M}+1)$; HRMS $(\mathrm{m} / z)$ calcd for $\mathrm{C}_{23} \mathrm{H}_{21} \mathrm{NO}_{7}: 441.1656[\mathrm{M}$ $\left.+\mathrm{NH}_{4}\right]^{+}$, Found: $441.1652\left[\mathrm{M}+\mathrm{NH}_{4}\right]^{+}$.

\subsection{General procedure for synthesis of $20 \mathrm{a}-\mathrm{j}$}

To a solution of aldehyde/ketone $(0.13 \mathrm{mmol})$ in $\mathrm{MeOH}(10 \mathrm{~mL})$ was added stable nitroxide radical acid $(0.13 \mathrm{mmol})$ and $4 \beta$-isocyanopodophyllotoxin $(11)(0.10 \mathrm{mmol})$ at room temperature. The reaction mixture was subsequently heated at reflux for $5 \mathrm{~h}$. Upon completion of the reaction (TLC monitoring), the mixture was cooled to room temperature and solvent was evaporated. The residue was purified by chromatography on silica gel using EtOAc/petroleum ether as eluant to give 12a-j.

4.3.1. Compound 12a-Yield 36\%; m.p: $83-85^{\circ} \mathrm{C}$; IR (KBr) cm ${ }^{-1}$ : $3431(\mathrm{~N}-\mathrm{H}), 1776$, $1717(\mathrm{C}=\mathrm{O}), 1589,1506,1484,1465(\mathrm{Ar}), 1383(\mathrm{~N}-\mathrm{O}), 931\left(\mathrm{OCH}_{2} \mathrm{O}\right)$; ESR $\left(1 \times 10^{-5} \mathrm{~mol} / \mathrm{L}\right.$ in $\mathrm{EtOH}): g_{\mathrm{o}}=2.0057, A_{\mathrm{N}}=14.9 \times 10^{-4}, \Delta H_{\mathrm{O}}=2.66 \times 10^{-4}$; MS (EI) $(\mathrm{m} / \mathrm{z}): 667[\mathrm{M}+2 \mathrm{H}]^{+}$; HRMS (ESI) $(\mathrm{m} / \mathrm{z})$ for $\mathrm{C}_{35} \mathrm{H}_{41} \mathrm{~N}_{2} \mathrm{O}_{11}[\mathrm{M}+\mathrm{Na}]^{+}$: calc. 688.2710, found 688.2723.

4.3.2. Compound 12b-Yield 52\%; m.p: $138-139^{\circ} \mathrm{C}$; IR $(\mathrm{KBr}) \mathrm{cm}^{-1}: 3433(\mathrm{~N}-\mathrm{H})$, 1778, $1723(\mathrm{C}=\mathrm{O}), 1589,1504,1485(\mathrm{Ar}), 1385(\mathrm{~N}-\mathrm{O}), 935\left(\mathrm{OCH}_{2} \mathrm{O}\right)$; ESR $\left(1 \times 10^{-5} \mathrm{~mol} / \mathrm{L}\right.$ in EtOH): $g_{0}=2.0057, A_{\mathrm{N}}=14.8 \times 10^{-4}, \Delta H_{\mathrm{O}}=2.61 \times 10^{-4}$; MS (EI) $(\mathrm{m} / \mathrm{z}): 736[\mathrm{M}+\mathrm{Na}]^{+}$; HRMS (ESI) $(m / z)$ for $\mathrm{C}_{39} \mathrm{H}_{41} \mathrm{~N}_{2} \mathrm{O}_{11}[\mathrm{M}+\mathrm{Na}]^{+}$: calc. 736.2710, found 736.2752.

4.3.3. Compound 12c-Yield 79\%; m.p: $113-115^{\circ} \mathrm{C}$; IR (KBr) $\mathrm{cm}^{-1}: 3437(\mathrm{~N}-\mathrm{H}), 1778$, $1720(\mathrm{C}=\mathrm{O}), 1588,1506,1484,1466(\mathrm{Ar}), 1383(\mathrm{~N}-\mathrm{O}), 932\left(\mathrm{OCH}_{2} \mathrm{O}\right)$; ESR $\left(1 \times 10^{-5} \mathrm{~mol} / \mathrm{L}\right.$ in EtOH): $g_{0}=2.0058, A_{\mathrm{N}}=14.7 \times 10^{-4}, \Delta H_{\mathrm{O}}=2.65 \times 10^{-4}$; MS (EI) $(\mathrm{m} / \mathrm{z}): 680[\mathrm{M}+\mathrm{H}]^{+}$; HRMS (ESI) $\left(\mathrm{m} / z\right.$ ) for $\mathrm{C}_{36} \mathrm{H}_{43} \mathrm{~N}_{2} \mathrm{O}_{11}[\mathrm{M}+\mathrm{H}]^{+}$: calc. 680.2867, found 680.2864.

4.3.4. Compound 12d-Yield 66\%; m.p: $119-121^{\circ} \mathrm{C}$; IR $(\mathrm{KBr}) \mathrm{cm}^{-1}: 3438(\mathrm{~N}-\mathrm{H})$, 1776, $1718(\mathrm{C}=\mathrm{O}), 1588,1505,1484(\mathrm{Ar}), 1382(\mathrm{~N}-\mathrm{O}), 932\left(\mathrm{OCH}_{2} \mathrm{O}\right)$; ESR $\left(1 \times 10^{-5} \mathrm{~mol} / \mathrm{L}\right.$ in EtOH): $g_{0}=2.0058, A_{\mathrm{N}}=14.9 \times 10^{-4}, \Delta H_{\mathrm{o}}=2.66 \times 10^{-4}$; MS (EI) $(\mathrm{m} / \mathrm{z}): 706[\mathrm{M}+\mathrm{H}]^{+} ; \mathrm{HRMS}$ $(\mathrm{ESI})(\mathrm{m} / z)$ for $\mathrm{C}_{38} \mathrm{H}_{45} \mathrm{~N}_{2} \mathrm{O}_{11}[\mathrm{M}+\mathrm{H}]^{+}$: calc. 706.3023 , found 706.3021 .

4.3.5. Compound 12e-Yield 48\%; m.p: $95-97^{\circ} \mathrm{C}$; IR (KBr) cm-: $3431(\mathrm{~N}-\mathrm{H}), 1774$, $1718(\mathrm{C}=\mathrm{O}), 1610,1508,1458(\mathrm{Ar}), 1364(\mathrm{~N}-\mathrm{O}), 932\left(\mathrm{OCH}_{2} \mathrm{O}\right)$; ESR $\left(1 \times 10^{-5} \mathrm{~mol} / \mathrm{L}\right.$ in EtOH): $g_{\mathrm{o}}=2.0057, A_{\mathrm{N}}=14.7 \times 10^{-4}, \Delta H_{\mathrm{o}}=2.61 \times 10^{-4}$; MS (EI) $(\mathrm{m} / \mathrm{z}): 744[\mathrm{M}+\mathrm{H}]^{+} ; \mathrm{HRMS}$ (ESI) $(\mathrm{m} / z)$ for $\mathrm{C}_{40} \mathrm{H}_{43} \mathrm{~N}_{2} \mathrm{O}_{12}[\mathrm{M}+\mathrm{H}]^{+}$: calc. 744.2816, found 744.2815.

4.3.6. Compound 12f-Yield 64\%; m.p: $105-107^{\circ} \mathrm{C}$; IR (KBr) $\mathrm{cm}^{-1}$ : $3428(\mathrm{~N}-\mathrm{H}), 1775$, $1719(\mathrm{C}=\mathrm{O}), 1588,1506,1456(\mathrm{Ar}), 1384(\mathrm{~N}-\mathrm{O}), 931\left(\mathrm{OCH}_{2} \mathrm{O}\right)$; ESR $\left(1 \times 10^{-5} \mathrm{~mol} / \mathrm{L}\right.$ in $\mathrm{EtOH}): g_{\mathrm{o}}=2.0057, A_{\mathrm{N}}=14.9 \times 10^{-4}, \Delta H_{\mathrm{o}}=2.61 \times 10^{-4}$; MS (EI) $(\mathrm{m} / z): 728[\mathrm{M}+\mathrm{H}]_{+} ; \mathrm{HRMS}$ (ESI) $(\mathrm{m} / \mathrm{z})$ for $\mathrm{C}_{40} \mathrm{H}_{43} \mathrm{~N}_{2} \mathrm{O}_{11}[\mathrm{M}+\mathrm{H}]^{+}$: calc. 728.2867 , found 728.2864 .

4.3.7. Compound 12g-Yield 62\%; m.p: $119-121^{\circ} \mathrm{C}$; IR $(\mathrm{KBr}) \mathrm{cm}^{-1}: 3438(\mathrm{~N}-\mathrm{H})$, 1776, $1719(\mathrm{C}=\mathrm{O}), 1589,1506,1466(\mathrm{Ar}), 1383(\mathrm{~N}-\mathrm{O}), 930\left(\mathrm{OCH}_{2} \mathrm{O}\right)$; ESR $\left(1 \times 10^{-5} \mathrm{~mol} / \mathrm{L}\right.$ in EtOH): $g_{\mathrm{o}}=2.0058, A_{\mathrm{N}}=14.9 \times 10^{-4}, \Delta H_{\mathrm{O}}=2.65 \times 10^{-4}$; MS (EI) $(\mathrm{m} / \mathrm{z}): 705[\mathrm{M}+2 \mathrm{H}]^{+}$; HRMS (ESI) $(\mathrm{m} / \mathrm{z})$ for $\mathrm{C}_{37} \mathrm{H}_{39} \mathrm{~N}_{2} \mathrm{O}_{12}[\mathrm{M}+\mathrm{Na}]^{+}$: calc. 726.2503, found 726.2511. 
4.3.8. Compound 12h-Yield 59\%; m.p: $110-112{ }^{\circ} \mathrm{C}$; IR $(\mathrm{KBr}) \mathrm{cm}^{-1}: 3423(\mathrm{~N}-\mathrm{H})$, 1774, $1718(\mathrm{C}=\mathrm{O}), 1589,1507,1458(\mathrm{Ar}), 1363(\mathrm{~N}-\mathrm{O}), 931\left(\mathrm{OCH}_{2} \mathrm{O}\right)$; ESR $\left(1 \times 10^{-5} \mathrm{~mol} / \mathrm{L}\right.$ in EtOH): $g_{0}=2.0058, A_{\mathrm{N}}=14.7 \times 10^{-4}, \Delta H_{\mathrm{o}}=2.61 \times 10^{-4}$; MS (EI) $(\mathrm{m} / \mathrm{z}): 758[\mathrm{M}+\mathrm{H}]^{+} ; \mathrm{HRMS}$ (ESI) $(\mathrm{m} / z)$ for $\mathrm{C}_{40} \mathrm{H}_{41} \mathrm{~N}_{2} \mathrm{O}_{13}[\mathrm{M}+\mathrm{H}]^{+}$: calc. 758.2609, found 758.2611.

4.3.9. Compound 12i-Yield 85\%; m.p:110-112 ${ }^{\circ} \mathrm{C}$; IR (KBr) $\mathrm{cm}^{-1}: 3436(\mathrm{~N}-\mathrm{H}), 1778$, $1736(\mathrm{C}=\mathrm{O}), 1588,1506,1465(\mathrm{Ar}), 1364(\mathrm{~N}-\mathrm{O}), 933\left(\mathrm{OCH}_{2} \mathrm{O}\right)$; ESR $\left(1 \times 10^{-5} \mathrm{~mol} / \mathrm{L}\right.$ in $\mathrm{EtOH}): g_{0}=2.0057, A_{\mathrm{N}}=14.9 \times 10^{-4}, \Delta H_{\mathrm{o}}=2.65 \times 10^{-4} ; \mathrm{MS}(\mathrm{EI})(\mathrm{m} / \mathrm{z}): 697[\mathrm{M}+2 \mathrm{H}]^{+} ; \mathrm{HRMS}$ (ESI) $\left(\mathrm{m} / \mathrm{z}\right.$ ) for $\mathrm{C}_{37} \mathrm{H}_{47} \mathrm{~N}_{2} \mathrm{O}_{11}[\mathrm{M}+\mathrm{Na}]^{+}$: calc. 718.3180 , found 718.3185 .

4.3.10. Compound 12j-Yield 76\%; m.p: $106-108{ }^{\circ} \mathrm{C}$; IR (KBr) $\mathrm{cm}^{-1}: 3437(\mathrm{~N}-\mathrm{H})$, 1778, $1718(\mathrm{C}=\mathrm{O}), 1588,1506,1466(\mathrm{Ar}), 1362(\mathrm{~N}-\mathrm{O}), 934\left(\mathrm{OCH}_{2} \mathrm{O}\right)$; ESR $\left(1 \times 10^{-5} \mathrm{~mol} / \mathrm{L}\right.$ in EtOH): $g_{0}=2.0058, A_{\mathrm{N}}=14.7 \times 10^{-4}, \Delta H_{\mathrm{O}}=2.67 \times 10^{-4}$; MS (EI) $(\mathrm{m} / \mathrm{z}): 694[\mathrm{M}+\mathrm{H}]^{+}$; HRMS (ESI) $(\mathrm{m} / z)$ for $\mathrm{C}_{37} \mathrm{H}_{45} \mathrm{~N}_{2} \mathrm{O}_{11}[\mathrm{M}+\mathrm{H}]^{+}$: calc. 694.3023, found 694.3014 .

\subsection{General procedure for synthesis of $13 a-I$}

Aldehyde/ketone $(0.23 \mathrm{mmol})$, stable nitroxide radical acid $(0.23 \mathrm{mmol})$, and $4 \beta$ isocyanopodophyllotoxin $(\mathbf{1 1})(0.18 \mathrm{mmol})$ were added sequentially to a solution of amine $(0.23 \mathrm{mmol})$ in $\mathrm{MeOH}(10 \mathrm{~mL})$ at room temperature. The reaction mixture was heated at reflux for $5 \mathrm{~h}$. Upon completion of the reaction (TLC monitoring), the mixture cooled to room temperature and solvent was evaporated. The crude reaction mixture was purified by column chromatography (EtOAc/petroleum ether) to give the target product 13a-l.

4.4.1. Compound 13a-Yield 54\%; m.p: $120-123{ }^{\circ} \mathrm{C}$; IR (KBr) $\mathrm{cm}^{-1}: 3448(\mathrm{~N}-\mathrm{H}), 1772$ $(\mathrm{C}=\mathrm{O}), 1592,1507,1465(\mathrm{Ar}), 1377(\mathrm{~N}-\mathrm{O}), 934\left(\mathrm{OCH}_{2} \mathrm{O}\right)$; $\mathrm{ESR}\left(1 \times 10^{-5} \mathrm{~mol} / \mathrm{L}\right.$ in $\left.\mathrm{EtOH}\right)$ : $g_{0}=2.0058, A_{\mathrm{N}}=14.8 \times 10^{-4}, \Delta H_{\mathrm{o}}=2.66 \times 10^{-4}$; MS (EI) $(\mathrm{m} / z): 762[\mathrm{M}+2 \mathrm{H}]^{+} ;$HRMS (ESI) $(\mathrm{m} /$ z) for $\mathrm{C}_{40} \mathrm{H}_{54} \mathrm{~N}_{3} \mathrm{O}_{10}[\mathrm{M}+\mathrm{Na}]^{+}$: calc.783.3809, found 783.3806 .

4.4.2. Compound 13b-yield 47\%; m.p: $103-105^{\circ} \mathrm{C}$; IR (KBr) cm $\mathrm{cm}^{-1}$ : $3421(\mathrm{~N}-\mathrm{H}), 1775$, $1718(\mathrm{C}=\mathrm{O}), 1591,1507,1465(\mathrm{Ar}), 1358(\mathrm{~N}-\mathrm{O}), 931\left(\mathrm{OCH}_{2} \mathrm{O}\right)$; ESR $\left(1 \times 10^{-5} \mathrm{~mol} / \mathrm{L}\right.$ in $\mathrm{EtOH}): g_{0}=2.0057, A_{\mathrm{N}}=14.8 \times 10^{-4}, \Delta H_{\mathrm{O}}=2.65 \times 10^{-4}$; MS (EI) $(\mathrm{m} / z): 755[\mathrm{M}+\mathrm{H}]^{+} ; \mathrm{HRMS}$ (ESI) $(\mathrm{m} / z)$ for $\mathrm{C}_{42} \mathrm{H}_{48} \mathrm{~N}_{3} \mathrm{O}_{10}[\mathrm{M}+\mathrm{H}]^{+}$: calc.755.3340, found 755.3343.

4.4.3. Compound 13c-Yield 42\%; m.p: $157-159^{\circ} \mathrm{C}$; IR (KBr) $\mathrm{cm}^{-1}$ : $3423(\mathrm{~N}-\mathrm{H}), 1777$, $1735(\mathrm{C}=\mathrm{O}), 1592,1505,1458(\mathrm{Ar}), 1368(\mathrm{~N}-\mathrm{O}), 932\left(\mathrm{OCH}_{2} \mathrm{O}\right)$; ESR $\left(1 \times 10^{-5} \mathrm{~mol} / \mathrm{L}\right.$ in EtOH): $g_{0}=2.0057, A_{\mathrm{N}}=14.8 \times 10^{-4}, \Delta H_{\mathrm{O}}=2.65 \times 10^{-4}$; MS (EI) $(\mathrm{m} / z): 805[\mathrm{M}+\mathrm{H}]^{+} ; \mathrm{HRMS}$ (ESI) $(\mathrm{m} / \mathrm{z})$ for $\mathrm{C}_{46} \mathrm{H}_{50} \mathrm{~N}_{3} \mathrm{O}_{10}[\mathrm{M}+\mathrm{H}]^{+}$: calc. 805.3496, found 805.3497.

4.4.4. Compound 13d-Yield 50\%; m.p: $115-117^{\circ} \mathrm{C}$; IR (KBr) cm ${ }^{-1}: 3433(\mathrm{~N}-\mathrm{H}), 1772$ $(\mathrm{C}=\mathrm{O}), 1590,1506,1483(\mathrm{Ar}), 1370(\mathrm{~N}-\mathrm{O}), 934\left(\mathrm{OCH}_{2} \mathrm{O}\right)$; ESR $\left(1 \times 10^{-5} \mathrm{~mol} / \mathrm{L}\right.$ in $\left.\mathrm{EtOH}\right)$ : $g_{0}=2.0057, A_{\mathrm{N}}=14.9 \times 10^{-4}, \Delta H_{\mathrm{o}}=2.65 \times 10^{-4}$; MS (EI) $(\mathrm{m} / z): 708[\mathrm{M}+2 \mathrm{H}]^{+}$; HRMS (ESI) $(\mathrm{m} /$ z) for $\mathrm{C}_{38} \mathrm{H}_{48} \mathrm{~N}_{3} \mathrm{O}_{10}[\mathrm{M}+\mathrm{K}]^{+}$: calc. 745.3340, found 745.3342.

4.4.5. Compound 13e-Yied $42 \%$; m.p: $115-116^{\circ} \mathrm{C}$; IR $(\mathrm{KBr}) \mathrm{cm}^{-1}: 3429(\mathrm{~N}-\mathrm{H}), 1590$, 1507, $1483(\mathrm{Ar}), 1363(\mathrm{~N}-\mathrm{O}), 934\left(\mathrm{OCH}_{2} \mathrm{O}\right)$; $\mathrm{ESR}\left(1 \times 10^{-5} \mathrm{~mol} / \mathrm{L}\right.$ in $\left.\mathrm{EtOH}\right): g_{0}=2.0058$, $A_{\mathrm{N}}=14.7 \times 10^{-4}, \Delta H_{\mathrm{o}}=2.61 \times 10^{-4}$; MS (EI) $(\mathrm{m} / \mathrm{z}): 721[\mathrm{M}+\mathrm{H}]^{+}$; HRMS (ESI) $(\mathrm{m} / \mathrm{z})$ for $\mathrm{C}_{39} \mathrm{H}_{50} \mathrm{~N}_{3} \mathrm{O}_{10}[\mathrm{M}+\mathrm{H}]^{+}$: calc. 721.3496 , found 721.3493 .

4.4.6. Compound 13f-Yield 68\%; m.p: 86-88 ${ }^{\circ} \mathrm{C}$; IR $(\mathrm{KBr}) \mathrm{cm}^{-1}: 3434(\mathrm{~N}-\mathrm{H}), 1772$ $(\mathrm{C}=\mathrm{O}), 1592,1507,1466(\mathrm{Ar}), 1368(\mathrm{~N}-\mathrm{O}), 935\left(\mathrm{OCH}_{2} \mathrm{O}\right)$; ESR $\left(1 \times 10^{-5} \mathrm{~mol} / \mathrm{L}\right.$ in $\left.\mathrm{EtOH}\right)$ : $g_{0}=2.0057, A_{\mathrm{N}}=14.9 \times 10^{-4}, \Delta H_{\mathrm{o}}=2.65 \times 10^{-4}$; MS (EI) $(\mathrm{m} / \mathrm{z}): 722[\mathrm{M}+2 \mathrm{H}]^{+}$; HRMS (ESI) $(\mathrm{m} /$ z) for $\mathrm{C}_{39} \mathrm{H}_{50} \mathrm{~N}_{3} \mathrm{O}_{10}[\mathrm{M}+\mathrm{Na}]^{+}$: calc.743.3496, found 743.3492. 
4.4.7. Compound 13g-Yield 84\%; m.p: $147-149^{\circ} \mathrm{C}$; IR (KBr) cm ${ }^{-1}$ : $3426(\mathrm{~N}-\mathrm{H}), 1775$ $(\mathrm{C}=\mathrm{O}), 1507,1458(\mathrm{Ar}), 1368(\mathrm{~N}-\mathrm{O}), 932\left(\mathrm{OCH}_{2} \mathrm{O}\right)$; ESR $\left(1 \times 10^{-5} \mathrm{~mol} / \mathrm{L}\right.$ in $\left.\mathrm{EtOH}\right)$ : $g_{0}=2.0057, A_{\mathrm{N}}=14.8 \times 10^{-4}, \Delta H_{\mathrm{O}}=2.66 \times 10^{-4} ; \mathrm{MS}(\mathrm{EI})(\mathrm{m} / \mathrm{z}): 755[\mathrm{M}+\mathrm{H}]^{+} ; \mathrm{HRMS}(\mathrm{ESI})(\mathrm{m} /$ z) for $\mathrm{C}_{42} \mathrm{H}_{48} \mathrm{~N}_{3} \mathrm{O}_{10}[\mathrm{M}+\mathrm{H}]^{+}$: calc. 755.3340, found 755.3342.

4.4.8. Compound 13h-Yield 60\%; m.p: $95-96^{\circ} \mathrm{C}$; IR (KBr) $\mathrm{cm}^{-1}: 3392(\mathrm{~N}-\mathrm{H}), 1779$, $1717(\mathrm{C}=\mathrm{O}), 1609,1508,1451(\mathrm{Ar}), 1382(\mathrm{~N}-\mathrm{O}), 930\left(\mathrm{OCH}_{2} \mathrm{O}\right)$; ESR $\left(1 \times 10^{-5} \mathrm{~mol} / \mathrm{L}\right.$ in $\mathrm{EtOH}): g_{0}=2.0058, A_{\mathrm{N}}=14.7 \times 10^{-4}, \Delta H_{\mathrm{O}}=2.65 \times 10^{-4}$; MS (EI) $(\mathrm{m} / \mathrm{z}): 807[\mathrm{M}+\mathrm{Na}]^{+}$; HRMS (ESI) $(\mathrm{m} / \mathrm{z})$ for $\mathrm{C}_{43} \mathrm{H}_{50} \mathrm{~N}_{3} \mathrm{O}_{11}[\mathrm{M}+\mathrm{Na}]^{+}$: calc. 807.3445, found 807.3443.

4.4.9. Compound 13i-Yield 76\%; m.p: $106-109^{\circ} \mathrm{C}$; $\mathrm{IR}(\mathrm{KBr}) \mathrm{cm}^{-1}: 3426(\mathrm{~N}-\mathrm{H}), 1768$ $(\mathrm{C}=\mathrm{O}), 1591,1506(\mathrm{Ar}), 1374(\mathrm{~N}-\mathrm{O}), 934\left(\mathrm{OCH}_{2} \mathrm{O}\right)$; ESR $\left(1 \times 10^{-5} \mathrm{~mol} / \mathrm{L}\right.$ in $\left.\mathrm{EtOH}\right)$ : $g_{0}=2.0058, A_{\mathrm{N}}=14.7 \times 10^{-4}, \Delta H_{\mathrm{O}}=2.65 \times 10^{-4} ; \mathrm{MS}(\mathrm{EI})(\mathrm{m} / \mathrm{z}): 746[\mathrm{M}]^{+} ; \operatorname{HRMS}(\mathrm{ESI})(\mathrm{m} / \mathrm{z})$ for $\mathrm{C}_{41} \mathrm{H}_{52} \mathrm{~N}_{3} \mathrm{O}_{10}[\mathrm{M}]^{+}$: calc.746.3653, found 746.3655 .

4.4.10. Compound 13j-Yield 45\%; m.p: $110-112^{\circ} \mathrm{C}$; IR (KBr) cm ${ }^{-1}: 3447$ (N-H), 1772 $(\mathrm{C}=\mathrm{O}), 1590,1507,1466(\mathrm{Ar}), 1375(\mathrm{~N}-\mathrm{O}), 932\left(\mathrm{OCH}_{2} \mathrm{O}\right)$; ESR $\left(1 \times 10^{-5} \mathrm{~mol} / \mathrm{L}\right.$ in $\left.\mathrm{EtOH}\right)$ : $g_{0}=2.0058, A_{\mathrm{N}}=14.9 \times 10^{-4}, \Delta H_{\mathrm{o}}=2.66 \times 10^{-4} ; \mathrm{MS}(\mathrm{EI})(\mathrm{m} / \mathrm{z}): 736[\mathrm{M}+2 \mathrm{H}]^{+} ; \mathrm{HRMS}(\mathrm{ESI})(\mathrm{m} /$ z) for $\mathrm{C}_{40} \mathrm{H}_{52} \mathrm{~N}_{3} \mathrm{O}_{10}[\mathrm{M}+\mathrm{Na}]^{+}$: calc.757.3653, found 757.3652 .

4.4.11. Compound 13k-Yield 39\%; m.p: $86-89^{\circ} \mathrm{C}$; IR (KBr) $\mathrm{cm}^{-1}: 3432(\mathrm{~N}-\mathrm{H}), 1772$ $(\mathrm{C}=\mathrm{O}), 1591,1507,1465(\mathrm{Ar}), 1378(\mathrm{~N}-\mathrm{O}), 935\left(\mathrm{OCH}_{2} \mathrm{O}\right)$; $\mathrm{ESR}\left(1 \times 10^{-5} \mathrm{~mol} / \mathrm{L}\right.$ in $\left.\mathrm{EtOH}\right)$ : $g_{0}=2.0057, A_{\mathrm{N}}=14.8 \times 10^{-4}, \Delta H_{\mathrm{o}}=2.61 \times 10^{-4}$; MS (EI) $(\mathrm{m} / \mathrm{z}): 736[\mathrm{M}+2 \mathrm{H}]^{+}$; HRMS (ESI) $(\mathrm{m} /$ z) for $\mathrm{C}_{40} \mathrm{H}_{52} \mathrm{~N}_{3} \mathrm{O}_{10}[\mathrm{M}+\mathrm{Na}]^{+}$: calc. 757.3653 , found 757.3652 .

4.4.12. Compound 13I-Yield 46\%; m.p: $92-94^{\circ} \mathrm{C}$; $\mathrm{IR}(\mathrm{KBr}) \mathrm{cm}^{-1}: 3356(\mathrm{~N}-\mathrm{H}), 1778$ $(\mathrm{C}=\mathrm{O}), 1585,1451(\mathrm{Ar}), 1381(\mathrm{~N}-\mathrm{O}), 933\left(\mathrm{OCH}_{2} \mathrm{O}\right) ; \mathrm{ESR}\left(1 \times 10^{-5} \mathrm{~mol} / \mathrm{L}\right.$ in $\left.\mathrm{EtOH}\right)$ : $g_{0}=2.0057, A_{\mathrm{N}}=14.9 \times 10^{-4}, \Delta H_{\mathrm{o}}=2.66 \times 10^{-4}$; MS (EI) $(\mathrm{m} / \mathrm{z}): 738[\mathrm{M}+2 \mathrm{H}]^{+}$; HRMS (ESI) $(\mathrm{m} /$ z) for $\mathrm{C}_{40} \mathrm{H}_{54} \mathrm{~N}_{3} \mathrm{O}_{10}[\mathrm{M}+2 \mathrm{H}]^{+}$: calc.738.3809, found 738.3811 .

\subsection{Cytotoxicity assays}

Cytotoxic activity was determined by the sulforhodamine B (SRB) colorimetric assay as previously described [33]. In brief, the cells $\left(3-5 \times 10^{3}\right.$ cells/well) were seeded in 96-well plates filled with RPMI-1640 medium supplemented with $10 \%$ fetal bovine serum (FBS) containing various concentrations of samples, and incubated for $72 \mathrm{~h}$. At the end of the exposure period, the attached cells were fixed with cold 50\% trichloroacetic acid for $30 \mathrm{~min}$ followed by staining with $0.04 \%$ SRB (Sigma Chemical Co.) for $30 \mathrm{~min}$. The bound SRB was solubilized in $10 \mathrm{mM}$ Tris-base and the absorbance was measured at $515 \mathrm{~nm}$ on a Microplate Reader ELx800 (Bio-Tek Instruments, Winooski, VT) with a Gen5 software. All results were representative of three or more experiments.

\section{Acknowledgments}

This work was supported financially by the National Natural Science Foundation of China (30800720, 31371975); Partial financial support was supplied by Xinjiang Production \& Construction Corps Key Laboratory of Protection and Utilization of Biological Resources in Tarim Basin (BYRB1306); the Fundamental Research Funds for the Central Universities (lzujbky-2013-69) and the Young Scholars Science Foundation of Lanzhou Jiaotong University (2011011). Partial support was also supplied by NIH grant CA177584 from the National Cancer Institute awarded to K.H. Lee. Thanks are also due to the support of Taiwan Department of Health Cancer Research Center of Excellence (DOH-100-TD-C-111-005).

\section{References}

1. Dömling A. Chem. Rev. 2006; 106:17-89. [PubMed: 16402771] 
2. Akritopoulou-Zanze I. Curr. Opin. Chem. Biol. 2008; 12:324-331. [PubMed: 18312861]

3. Colombo M, Peretto I. Drug Discov. Today. 2008; 13:677-684. [PubMed: 18675762]

4. Sk UH, Dixit D, Sen E. Europ. J. Med. Chem. 2013; 68:47-57.

5. Ma Y, Fang S, Li H, Han C, Lu Y, Zhao Y, Liu Y, Zhao C. Chem. Biol. Drug Des. 2013; 82:12-21. [PubMed: 23786349]

6. Stahelin H, von Wartburg A. Prog. Drug Res. 1989; 33:169-266. [PubMed: 2687938]

7. Liu YQ, Yang L, Tian X. Curr. Bioact. Compds. 2007; 3:37-66.

8. Zhang YL, Tropsha A, McPhail AT, Lee KH. J. Med. Chem. 1994; 37:1460-1464. [PubMed: 8182704]

9. Huang TS, Lee CC, Chao Y, Shu CH, Chen LT, Chen LL, Chen MH, Yuan CC, Peng W. J. Pharm. Res. 1999; 16:997-1002.

10. Byl JAW, Cline SD, Utsugi T, Kobunai T, Yamada Y, Osheroff N. Biochemistry. 2001; 40:712718. [PubMed: 11170388]

11. Wang YG, Tian X, Chen YZ. Acta. Pharm. Sin. 1988; 23:792-799.

12. Chen YZ, Zhang CJ, Tian X. Scientia Sinica (series B). 1987; 30:1070-1079.

13. Chen YZ, Tian X, Wang YG. Life Sci. 1989; 45:2569-2575. [PubMed: 2615556]

14. Wang JZ, Tian X, Tsumura H. Anti-Cancer Drug Des. 1993; 8:193-202.

15. Tian X, Yan ZQ, Li JX, Chen YZ. Chem. Res. Chin. Univ. 1995; 11:79-83.

16. Tian X, Yang MG, Chen YZ. Chem. Res. Chin. Univ. 1996; 12:304-308.

17. Wang YG, Pan JL, Shi JF, Chen YZ. Life Sci. 1997; 61:537-542. [PubMed: 9247323]

18. Tian X, Wang YG, Yang MG, Chen YZ. Life Sci. 1997; 60:511-517. [PubMed: 9042385]

19. Jin Y, Chen SW, Tian X. Bioorg. Med. Chem. 2006; 14:3062-3068. [PubMed: 16406792]

20. Tian X, Zhang FM, Li WG. Life Sci. 2002; 70:2433-2443. [PubMed: 12150207]

21. Liu YQ, Tian X. Syn. Commun. 2005; 35:2749-2758.

22. Zhang JQ, Zhang ZW, Hui L, Chen SW, Tian X. Bioorg. Med. Chem. Lett. 2010; 20:983-986. [PubMed: 20053564]

23. Zhang ZW, Zhang JQ, Hui L, Chen SW, Tian X. Eur. J. Med. Chem. 2010; 45:1673-1677. [PubMed: 20071056]

24. Ye D, Shi Q, Leung CH, Kim SW, Park SY, Gullen EA, Jiang ZL, Zhu H, Morris-Natschke SL, Cheng YC, Lee KH. Bioorg Med Chem. 2012; 20:4489-4494. [PubMed: 22698783]

25. Liu JF, Sang CY, Xu XH, Zhang LL, Yang X, Hui L, Zhang JB, Chen SW. Europ. J. Med. Chem. 2013; 64:621-628.

26. Kamal A, Mallareddy A, Suresh P, Nayak VL, Shetti RVCRNC, Rao NS, Tamboli JR, Shaik TB, Vishnuvardhan MVPS, Ramakrishna S. Europ. J. Med. Chem. 2012; 47:530-545.

27. Andrés A, Jose L, Lopez-Perez E, Luis FG, Andrés F, Chiara T, Isabel B, Jose MA, Fernando D, Arturo SF. J. Med. Chem. 2012; 55:6724-67377. [PubMed: 22607205]

28. López-Pérez JL, del Olmo E, de Pascual-Teresa B, Abad A, San Feliciano A. Bioorg. Med. Chem. Lett. 2004; 14:1283-1286. [PubMed: 14980682]

29. Duca M, Guianvarch D, Meresse P, Bertounesque E, Dauzonne D, Kraus-Berthier L, Thirot S, Léonce S, Pierré A, Pfeiffer B, Renard P, Arimondo PB, Monneret C. J. Med. Chem. 2005; 48:593-603. [PubMed: 15658872]

30. Kamal A, Kumar BA, Suresh P, Juvekar A, Zingde S. Bioorg. Med. Chem. 2011; 19:2975-2979. [PubMed: 21489802]

31. Liu YQ, Li LH, Li WQ, Feng G, Yang L. Chem. Pap. 2011; 65:739-742.

32. Yu YP, Chen SY, Wang YG, Chen YZ. Tetrahedron Lett. 1999; 40:1967-1970.

33. Skehan P, Storeng R, Scudiero D, Monks A, McMahon J, Vistica D, Warren JT, Bokesch H, Kenney S, Boyd MR. J. Natl. Cancer Inst. 1990; 82:1107-1112. [PubMed: 2359136]

34. Xiao ZY, Bastow KF, Vance JRRS, Sidwell, Wang HK, Chen MS, Shi Q, Lee KH. J. Med. Chem. 2004; 47:5140-5148. [PubMed: 15456257]

35. Li WQ, Wang XL, Qian K, Liu YQ, Wang CY, Yang L, Tian J, Morris-Natschke SL, Zhou XW, Lee KH. Bioorg. Med. Chem. 2013; 21:2363-2369. [PubMed: 23490151] 
36. Zhang ZJ, Tian J, Wang LT, Wang MJ, Nan X, Yang L, Liu YQ, Morris-Natschke SL, Lee KH.

Bioorg Med Chem. 2014; 22:204-210. [PubMed: 24332656] 


\section{HIGHLIGHTS}

New spin-labeled podophyllotoxin analogs were prepared and tested for cytotoxicity.

The synthesis used an isocyanide multicomponent coupling reaction.

Potent cytotoxicity was found against A-549, DU-145, KB and KBvin cancer cell lines.

Two compounds (12e, 12h) showed superior potency to etoposide. 
<smiles>[R][R]</smiles>

1. Podophyllotoxin

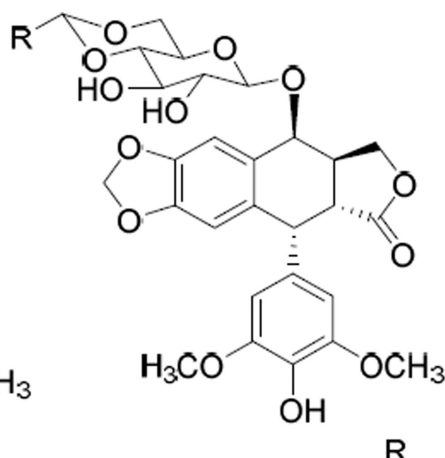

2. Etoposide $\frac{\mathrm{R}}{\mathrm{CH}_{3}}$

3. Teniposide 4<smiles></smiles>

4. NPF<smiles></smiles>

5. GL331<smiles>COc1cc([C@H]2c3cc4c(cc3C(CCNCCC(C)C)[C@H]3COC(=O)[C@@H]23)OCO4)cc(OC)c1O</smiles>

6. TOP-53<smiles>COc1ccc(C(OC(=O)C2=CC(C)(C)N(O)C2(C)C)C(=O)NC2c3cc4c(cc3[C@@H](c3cc(OC)c(OC)c(OC)c3)[C@H]3C(=O)OC[C@@H]23)OCO4)cc1</smiles>

$12 \mathrm{e}$<smiles></smiles>

7. GP-7<smiles>COc1cc([C@H]2c3cc4c(cc3[C@@H](O)C(CO)[C@@H]2C(=O)NNC(=O)NC2=CC(C)(C)N(O)C2(C)C)OCO4)cc(OC)c1OC</smiles><smiles>COc1cc([C@H]2c3cc4c(cc3[C@@H](NC(=O)C(OC(=O)C3=CC(C)(C)N(O)C3(C)C)c3ccc5c(c3)OCO5)[C@H]3COC(=O)[C@H]23)OCO4)cc(OC)c1OC</smiles>

$12 \mathrm{~h}$<smiles>COc1cc([C@H]2c3cc4c(cc3C(NC(=O)C(c3ccccc3)N(C(=O)C3CC(C)(C)N([O])C(C)(C)C3)c3ccccc3)[C@@H]3COC(=O)[C@H]23)OCO4)cc(OC)c1OC</smiles>

$13 c$

Fig. 1.

Structures of podophyllotoxin derivatives. 


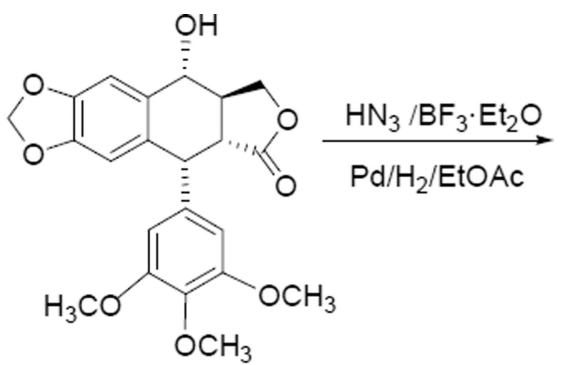

1

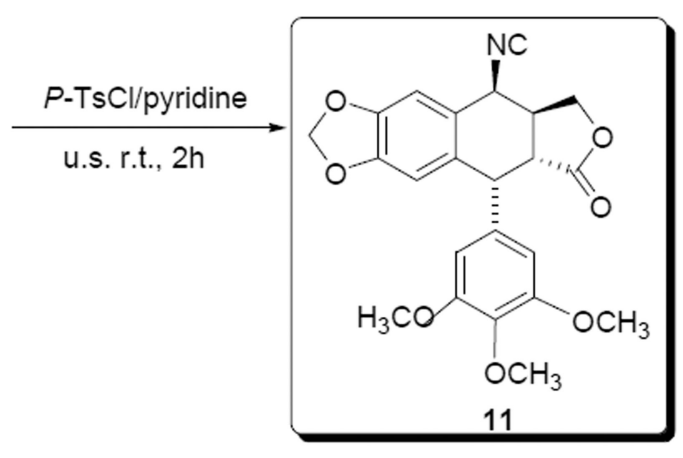

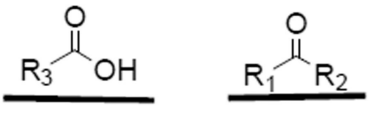<smiles>COc1cc([C@H]2c3cc4c(cc3[C@@H](N)[C@@H]3COC(=O)[C@H]23)OCO4)cc(OC)c1OC</smiles>

9

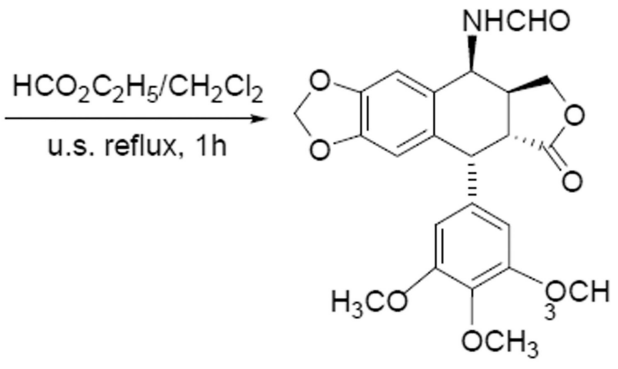

10

12a-j

$12 a$<smiles>CC(=O)CCCCCCCCCCCC(C)=O</smiles>

$12 d$<smiles>CC1(C)C=C(C(=O)O)C(C)(C)N1[O]</smiles>
$12 b$

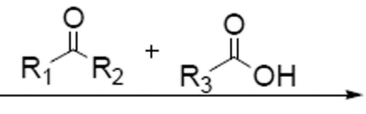
$\mathrm{MeOH}$, reflux, $5 \mathrm{~h}$.

Passirini reaction<smiles>[R3]C(=O)OC([R])([R])C(=O)N[C@H]1c2cc3c(cc2[C@H](c2cc(OC)c(OC)c(OC)c2)[C@H]2C(=O)OC[C@H]12)OCO3</smiles>

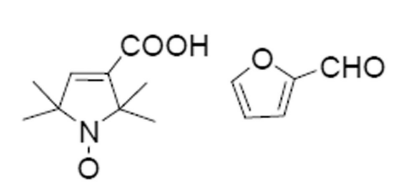<smiles>CC1(C)C=C(C(=O)O)C(C)(C)N1[O]</smiles><smiles>O=Cc1ccccc1</smiles><smiles>[Mg]</smiles><smiles>CC1(C)C=C(C(=O)O)C(C)(C)N1[O]</smiles>
$\mathrm{CH}_{3}\left(\mathrm{CH}_{2}\right)_{2} \mathrm{CHO}$

$12 \mathrm{e}$<smiles>CC1(C)C=C(C(=O)[O-])C(C)(C)N1O</smiles><smiles>COc1ccc(C=O)cc1</smiles>
$12 f$<smiles>CC1(C)C=C(C(=O)[O-])C(C)(C)N1O</smiles><smiles>O=CCc1ccccc1</smiles>
$12 \mathrm{~g}$<smiles>CCCCCCCCCCCC(=O)O</smiles>

Scheme 1.

Synthesis of target compounds $\mathbf{1 2} \mathbf{a}-\mathbf{j}$ via the Passerini reaction. 
<smiles>COc1cc([C@H]2c3cc4c(cc3C(C#N)C3COC(=O)[C@@H]32)OCO4)cc(OC)c1OC</smiles>

R $1-\mathrm{NH}_{2}$<smiles>[R]C([R])=O</smiles><smiles>[R]C(=O)O</smiles>

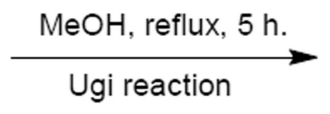
$\mathrm{R}_{4} \mathrm{OH}$<smiles>[R]C(=O)CCCCCC([R1])=O</smiles>

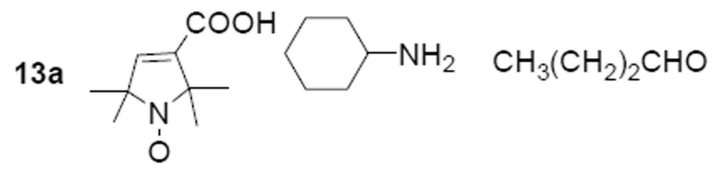<smiles>CC1(C)C=C(C(=O)O)C(C)(C)N1C=O</smiles><smiles>Nc1ccccc1</smiles>
$\mathrm{CH}_{3}\left(\mathrm{CH}_{2}\right)_{2} \mathrm{CHO}$ $13 c$<smiles>CC1(C)CC(C(=O)O)CC(C)(C)N1O</smiles>

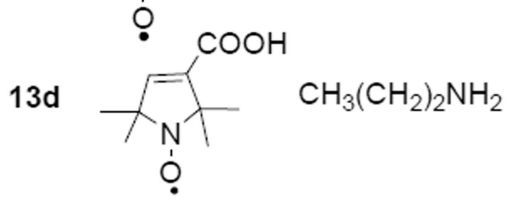<smiles>CC(C)=O</smiles><smiles>O=Cc1ccccc1</smiles>

$3 e$<smiles>CC1(C)C=C(C(=O)O)CC(C)(C)N1O</smiles>
$\mathrm{CH}_{3}\left(\mathrm{CH}_{2}\right)_{2} \mathrm{NH}_{2}$<smiles>CC(C)=O</smiles>

Scheme 2.

Synthesis of target compounds 13a-l via the Ugi reaction.<smiles>CCCNCCN</smiles><smiles>O=Cc1ccccc1</smiles>

$13 \mathrm{~h}$<smiles>CC1=C(C(=O)O)CC(C)(C)C1(C)O</smiles>
$\mathrm{CH}_{3}\left(\mathrm{CH}_{2}\right)_{2} \mathrm{NH}_{2} \quad \mathrm{H}_{3} \mathrm{CO}$<smiles>Cc1ccc(C=O)cc1</smiles>

$13 i$<smiles>CN1C(C)(C)C=C(C(=O)O)C1(C)C</smiles>
$\mathrm{CH}_{3}\left(\mathrm{CH}_{2}\right)_{2} \mathrm{NH}_{2}$<smiles>O=C1CCCCC1</smiles>

13j

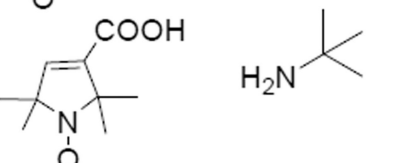
$\mathrm{CH}_{3}\left(\mathrm{CH}_{2}\right)_{2} \mathrm{CHO}$<smiles>CC1(C)C=C(C(=O)O)CC(C)(C)N1O</smiles>
$\mathrm{CH}_{3}\left(\mathrm{CH}_{2}\right)_{2} \mathrm{NH}_{2} \quad \mathrm{CH}_{3}\left(\mathrm{CH}_{2}\right)_{2} \mathrm{CHO}$

131<smiles>CN1C(C)(C)CC(C(=O)O)CC1(C)C</smiles>
$\mathrm{CH}_{3}\left(\mathrm{CH}_{2}\right)_{2} \mathrm{NH}_{2} \quad \mathrm{CH}_{3}\left(\mathrm{CH}_{2}\right)_{2} \mathrm{CHO}$ 
Table 1

In vitro cytotoxicity of compounds $\mathbf{1 2} \mathbf{a}-\mathbf{j}$ and $\mathbf{1 3 a}-\mathbf{c}$ against four human tumor cell lines ${ }^{a}$

\begin{tabular}{ccccc}
\hline \multirow{2}{*}{ Compd } & \multicolumn{4}{c}{$\mathbf{I C}_{\mathbf{5 0}}(\boldsymbol{\mu M})$} \\
\cline { 2 - 5 } & $\mathbf{A 5 4 9}$ & $\mathbf{D U 1 4 5}$ & $\mathbf{K B}$ & KBvin \\
\hline $\mathbf{1 2 a}$ & $2.38 \pm 0.27$ & $2.39 \pm 0.26$ & $2.32 \pm 0.12$ & $2.55 \pm 0.55$ \\
$\mathbf{1 2 b}$ & $>20$ & $>20$ & $>20$ & $>20$ \\
$\mathbf{1 2 c}$ & $8.86 \pm 0.43$ & $7.07 \pm 0.44$ & $7.12 \pm 0.45$ & $8.88 \pm 0.37$ \\
$\mathbf{1 2 d}$ & $9.52 \pm 0.82$ & $8.74 \pm 0.77$ & $9.24 \pm 1.57$ & $13.68 \pm 1.96$ \\
$\mathbf{1 2 e}$ & $0.75 \pm 0.02$ & $0.63 \pm 0.09$ & $0.60 \pm 0.02$ & $0.73 \pm 0.06$ \\
$\mathbf{1 2 f}$ & $7.68 \pm 0.37$ & $6.82 \pm 0.16$ & $6.53 \pm 0.06$ & $7.71 \pm 0.19$ \\
$\mathbf{1 2 g}$ & $7.38 \pm 0.04$ & $6.55 \pm 0.12$ & $7.12 \pm 0.22$ & $7.35 \pm 0.36$ \\
$\mathbf{1 2 h}$ & $1.46 \pm 0.17$ & $1.37 \pm 0.08$ & $1.12 \pm 0.13$ & $2.03 \pm 0.38$ \\
$\mathbf{1 2 i}$ & $8.82 \pm 0.26$ & $7.13 \pm 0.07$ & $6.58 \pm 0.14$ & $7.96 \pm 0.14$ \\
$\mathbf{1 2 j}$ & $9.05 \pm 0.26$ & $7.27 \pm 0.05$ & $6.88 \pm 0.24$ & $8.35 \pm 0.06$ \\
$\mathbf{1 3 a}$ & $8.98 \pm 1.21$ & $10.95 \pm 1.20$ & $9.35 \pm 2.01$ & $8.83 \pm 1.33$ \\
$\mathbf{1 3 b}$ & $8.40 \pm 0.304$ & $9.78 \pm 2.20$ & $8.49 \pm 0.72$ & $7.47 \pm 1.15$ \\
$\mathbf{1 3 c}$ & $6.10 \pm 0.192$ & $6.08 \pm 0.138$ & $5.94 \pm 0.37$ & $6.45 \pm 0.396$ \\
$\mathbf{2}$ & $2.58 \pm 0.252$ & $2.03 \pm 0.121$ & $3.88 \pm 0.199$ & $>20$ \\
\hline
\end{tabular}

${ }^{a}$ The results are average of three independent experiments. Compounds 13d-13I did not show significant inhibition (IC $\left.50>20 \mu \mathrm{M}\right)$. 